COUPLING IMPEDANCE AND ENERGY LOSS WITH MAGNET LAMINA'T IONS

R. Gluckstern

November 1985 


\section{COUPLING IMPEDANCE AND ENERGY LOSS}

\section{WITH MAGNET LAMINATIONS}

Robert Gluckstern*

November 1985

\section{Introduction}

The purpose of this note is to review the longitudinal coupling impedance for the Fermilab Booster. and to estimate the energy loss in the magnet laminations. The work is a review of earlier calculations by snowdon ${ }^{l}$ and Ruggiero. ${ }^{2}$

\section{Coupling Impedance}

The longitudinal coupling impedance of beam pipe has been calculated by many persons ${ }^{3}$. The result for a pipe wall of arbitrary surface impedance can be written ${ }^{4}$. for a beam radius $a$, and pipe radius $b$, as

$$
\left(z_{L} / n Z_{0}\right)=\left(2 i / s y^{2}\right)\left(\frac{1-2 \cdot F_{T}(x) I_{T}(x)}{x^{2}}\right)
$$

where

$$
x \equiv \sigma a, \quad \sigma \equiv(\omega / v \gamma), \quad n \equiv(R \omega / v)
$$

and where $I_{l}(x)$ is the usual modified Bessel Function.

$\star$ Permanent address: Physics Department, University of Maryland, College Park, MD 
The function $F_{1}(x)$ is

$$
F_{7}(x) \equiv K_{1}(x)+\alpha I_{7}(x)
$$

where $\alpha$ is determined by the boundary condition at the beam pipe. For a perfect conducting wall, one has

$$
\alpha=\frac{K_{0}(\sigma b)}{I_{0}(\sigma b)}
$$

leading to the wel1-known-result at large $\gamma(o b<<1)^{5}$

$$
\left(Z_{L}^{(0)} / n Z_{0}\right) \simeq\left(i / B \gamma^{2}\right)\left(\frac{1}{4}+\ln \frac{b}{a}\right)
$$

For a wall with small but finite wall impedance $z_{w}$ (defined as the ratio $-E_{z} / H_{\theta}$ ), one has

$$
\alpha \simeq \frac{K_{0}(\sigma b)}{I_{0}(\sigma b)}+i \frac{\beta^{2} \gamma^{2} c}{\omega b} \frac{Z_{w}}{Z_{0}}
$$

The corresponding coupling impedance is

$$
\left(Z_{L} / n Z_{0}\right) \simeq \frac{i}{B \gamma^{2}}\left(\frac{1}{4}+\ln \frac{b}{a}\right)+\frac{v}{\omega b} \frac{Z_{w}}{Z_{0}}
$$

For a smooth wall with finite conductivitiy, the wall impedance is given by 


$$
z_{w} / z_{0}=\frac{1-i}{2} \frac{\omega}{c} \delta
$$

so that the contribution to the coupling impedance is

$$
\left(\Delta Z_{L} / n Z_{0}\right)=\frac{1-i}{2} \quad \frac{B \delta}{b}
$$

\section{Wall Impedance}

If the wall consists of laminations of thickness D. permeability $\mu_{2}$, conductivity $\sigma_{2}$ corresponding to a skin depth $\delta$. separated by a dielectric of thickness $h$, permittivity $\varepsilon_{1}$. conductivity $\sigma_{1}$, the equivalent wall impedance can be calculated by treating the "crack". which extends from $r=b$ $\equiv \mathrm{b}_{1}$ to $r=\mathrm{b}_{2}$. as a radial transmission line. ${ }^{1.2}$ For the parameters appropriate to the Booster one can neglect losses in the dielectric, and consider the end of the transmission line to be a short. The geometry of the crack is as shown in the figure:

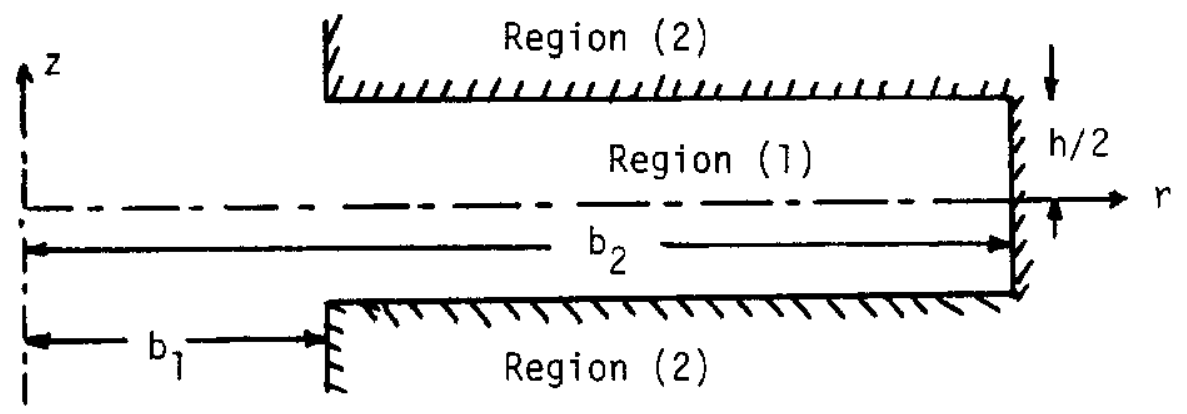

The field components for the azimuthally symmetric (outgoing) radial mode are given by 
Region (1) $|z|<h / 2$

$$
\begin{aligned}
& E_{z}=A \cosh \left(g_{7} z\right) H_{0}^{(1)}(k r) \\
& E_{r}=-\left(g_{T} A / k\right) \sinh \left(g_{T} z\right) H_{1}^{(1)}(k r) \\
& H_{\theta}=\left(-i \omega \varepsilon_{1} A / k\right) \cosh \left(g_{7} z\right) H_{1}^{(1)}(k r)
\end{aligned}
$$

\section{Region (2)}

$$
\begin{aligned}
& E_{z}=B e^{-g_{2}|z| H_{0}^{(1)}(k r)} \\
& E_{r}=\left(g_{2} B / k\right) e^{-g_{2}|z|_{H_{1}}^{(l)}(k r)} \\
& H_{\theta}=\left(\sigma_{2} B / k\right) \mathrm{e}^{-g_{2}|z|_{H_{1}}(l)}(k r)
\end{aligned}
$$

where

$$
\begin{aligned}
& k^{2}=k_{1}^{2}+g_{1}^{2}, \\
& k_{1}^{2}=\omega^{2} \mu_{1} \varepsilon_{1}, \\
& k^{2}=\left(2 i / \delta^{2}\right)+g_{2}^{2}
\end{aligned}
$$

For the parameters of interest

$$
\left|g_{2}\right|>k_{1}
$$


Continuity of $E_{r}$ and $H_{\theta}$ at $z=h / 2$ leads to

$$
g_{1}^{2} \simeq(1+i)\left(\mu_{2} / \mu_{1}\right) k_{1}{ }^{2}(\delta / h), \quad g_{2} \simeq \frac{1-i}{\delta}
$$

Assuming a short at $r=b_{2}$. we can write for the "crack" impedance

$$
\begin{aligned}
\frac{Z_{c}}{Z_{0}}=-\frac{E_{z}\left(r=b_{1}\right)}{Z_{0} H_{\theta}\left(r=b_{1}\right)} & =-\frac{i k c \varepsilon_{0}}{\varepsilon_{1} \omega} \frac{H_{0}^{(1)}\left(k b_{1}\right) H_{0}^{(2)}\left(k b_{2}\right)-H_{0}^{(2)}\left(k b_{1}\right) H_{0}^{(1)}\left(k b_{2}\right)}{H_{1}^{(1)}\left(k b_{1}\right) H_{0}^{(2)}\left(k b_{2}\right)-H_{1}^{(2)}\left(k b_{1}\right) H_{0}^{(1)}\left(k b_{2}\right)} \\
& =-\frac{i k c \varepsilon_{0}}{\omega \varepsilon_{1}} \frac{J_{0}\left(k b_{2}\right) N_{0}\left(k b_{1}\right)-J_{0}\left(k b_{1}\right) N_{0}\left(k b_{2}\right)}{J_{0}\left(k b_{2}\right) N_{1}\left(k b_{1}\right)-J_{1}\left(k b_{1}\right) N_{0}\left(k b_{2}\right)}
\end{aligned}
$$

For $\mu_{2} / \mu_{1} \simeq 100, \cdot 1<\delta / h<1$, we can write

$$
k^{2} \simeq g_{1}{ }^{2} \simeq(1+i) \frac{\mu_{2}}{\mu_{1}} \frac{\varepsilon_{1}}{\varepsilon_{0}} \frac{\omega^{2}}{c^{2}} \frac{\delta}{h}
$$


Equation (3.6) is very close to that obtalned by Ruggiero (FN-220. (17)), but he includes the factor $v / w b_{1}$ in Eq. (2.7) to convert wall impedance to coupling impedance, and also calibrates the impedance to the width of the "crack".

The low frequency limit of Eq. $(3.6)$ is

$$
\frac{z_{c}(\omega \rightarrow 0)}{z_{0}} \simeq(1-i) \frac{b_{1} \omega}{c} \frac{\delta}{h} \frac{\mu_{2}}{\mu_{0}} \ln \left(b_{2} / b_{1}\right)
$$

while the high frequency limit for $b_{2}>>b_{1}$ is

$$
\frac{Z_{c}(\omega+\infty)}{Z_{0}} \simeq-i \frac{k c \varepsilon_{0}}{\omega \varepsilon_{1}} \frac{H_{0}^{(1)}\left(k b_{1}\right)}{H_{1}^{(T)}\left(k b_{1}\right)} \simeq(1+i)^{\frac{1}{2}}\left(\frac{\mu_{2} \varepsilon_{0} \delta}{\mu_{0} \varepsilon_{1} h}\right)^{\frac{1}{2}}-i \frac{c \varepsilon_{0}}{2 b_{1} \omega \varepsilon_{1}}
$$

These results are consistent with the numerical results of snowdon, suggesting an asymptotic result varying as $w^{-1 / 4}$ with the value at $500 \mathrm{MHz}$

$$
\frac{Z_{c}(\omega / 2 \pi=500 \mathrm{MHz})}{Z_{0}} \simeq 1.4+i 0.3
$$

compared with that of Snowdon which is

$$
\left.\frac{z_{c}}{z_{0}} \simeq 1.57(\text { decreasing with } \omega)+i 0.24 \text { (increasing with } \omega\right)
$$

at $500 \mathrm{MHz}$. 
The contribution to the longitudinal coupling impedance can be obtained by weighting the "crack" impedance in Eqs. $(3.6),(3.8),(3.9)$ and the wall impedance in Eq. (2.8) proportional to the thickness of each. The justification for this is that the boundary does not disturb $\mathrm{H}_{\Theta}$ which causes compensating currents to flow in the laminations, but does disturb the electric field pattern in such a way that

$$
E_{z}{ }^{a v}=\frac{h E_{z}^{\text {"crack" }}+D E_{z}^{\text {"iron" }}}{h+D}
$$

leading to

$$
\frac{z_{\text {wall }}^{\text {equiv }}}{z_{0}} \simeq \frac{\frac{T-j}{2} \frac{\omega}{c} \delta 0+\left(z_{c} / z_{0}\right) h}{h+D}
$$

For $z_{c} / Z_{0} \gg w \delta / c, D>>h$, one has

$$
\frac{Z_{\text {wall }}}{Z_{0}} \simeq \frac{Z_{c} h}{Z_{0} \bar{D}} \bar{l}= \begin{cases}(1-i) \frac{b_{1} \omega}{c} \frac{\delta}{D_{0}} \frac{\mu_{2}}{\mu_{0}} \ln \left(b_{2} / b_{1}\right), & \text { low } \omega \\ (1+i)^{\frac{1}{2}}\left(\frac{\mu_{2}}{\mu_{0}} \frac{\varepsilon_{0}}{\varepsilon_{1}} \delta h\right)^{\frac{1}{2}} / 0, & \text { high } \omega\end{cases}
$$

The contribution to the coupling impedance is therefore 


$$
\frac{\Delta Z_{L}}{n Z_{0}}= \begin{cases}\hat{\alpha}(1-i) \beta \frac{\delta}{\bar{D}} \frac{\mu_{2}}{\mu_{0}} \ln \left(b_{2} / b_{1}\right) & \text { low } \omega \\ \hat{\alpha}(1+i)^{\frac{1}{2}} \frac{\beta c}{\omega b_{1}}\left(\frac{\mu_{2}}{\mu_{0}} \frac{\varepsilon_{0}}{\varepsilon_{1}} \delta h\right)^{\frac{1}{2} / D} & \text { high } \omega\end{cases}
$$

where $\hat{\alpha}$ is the fraction of the circumference occupied by the laminations.

The equivalent longitudinal coupling impedance per unit length is obtained by dividing $\Delta Z_{L}$ by $R Z_{0}$ giving

$$
\frac{\Delta Z_{L}}{R Z_{0}}= \begin{cases}\hat{\alpha}(1-i) \frac{\omega}{c} \frac{\delta}{D} \frac{\mu_{2}}{\mu_{0}} \ln \left(b_{2} / b_{1}\right) & \text { low } \omega \\ \hat{\alpha} \frac{(1+i)^{\frac{1}{2}}}{b_{1} D}\left(\frac{\mu_{2}}{\mu_{0}} \frac{\varepsilon_{0}}{\varepsilon_{1}} \delta h\right)^{\frac{1}{2}}, & \text { high } \omega\end{cases}
$$

[Equation (3.16) appears to correspond to Ruggiero's $Z_{n}$ (FN220. p. 9) but is a factor 2 smaller.] 
IV. Effect of a Coating

If the magnet is covered with a coating at the bore. the impedance seen by the beam will be modified.

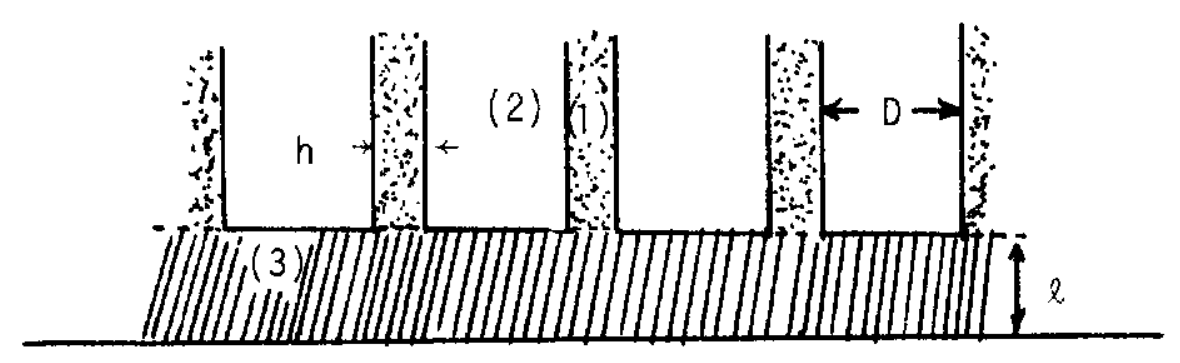

The details will depend on the relative size of $l, h, D$. In particular, if $\ell<h<<D$, one expects modification of the "crack" and "iron" impedances separately due to the coating. The combination will then be weighted as in Eq. (3.13). On the other hand, if $h>h$, one expects the coating to modify the equivalent wall impedance in Eqs. (3.13), (3.14) directly. In either event, it is necessary to calculate the impedance seen by the combination shown.

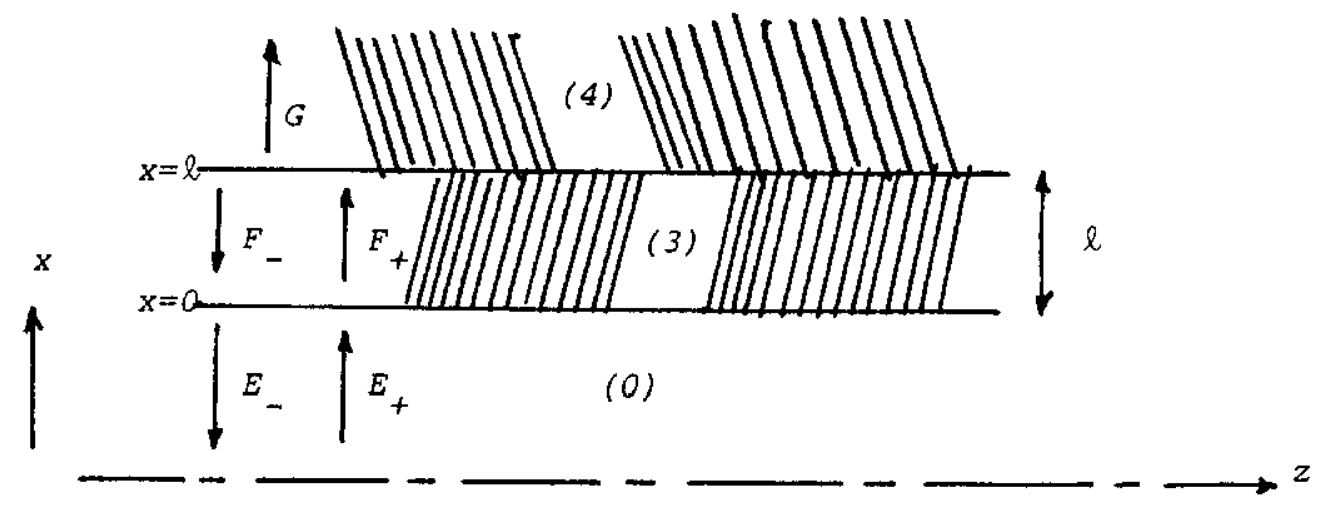


where the medium denoted by (4) is either the crack, the iron, or the combined equivalent. We will assume the validity of rectangular geometry, and will ignore variation with $z$, which takes place over distances much larger than $\ell$. For plane waves traveling in the $x$ direction, we have

$$
\begin{aligned}
E_{0} & =E_{+} e^{i k_{0} x}+E_{-} e^{-i k_{0} x} \\
Z_{0} H_{0} & =E_{+} e^{i k_{0} x}-E_{-} e^{-i k_{0} x} \\
E_{3} & =F_{+} e^{i k_{3} x}+F_{-} e^{-i k_{3} x} \\
Z_{3} H_{3} & =F_{+} e^{i k_{3} x}-F_{-} e^{-i k_{3} x} \\
E_{4} & =G e^{i k_{4}(x-l)} \\
Z_{4} H_{4} & =G e^{i k_{4}(x-l)}
\end{aligned}
$$

Continuity of $E$ and $H$ at the interfaces yields

$$
\begin{aligned}
& \frac{F_{+} e^{i k_{3} l}}{F_{-} e^{-i k_{3} l}}=\frac{Z_{4}+Z_{3}}{Z_{4}-Z_{3}} \\
& \frac{1-\frac{E_{0}}{Z_{3}}}{H_{0}}=\left.\frac{E_{3}}{Z_{3} H_{3}}\right|_{x=0}=\frac{F_{+}+F}{F_{+}-F_{-}}
\end{aligned}
$$


The equivalent impedance $Z$, seen at $x=0$ is therefore given by

$$
\begin{aligned}
\frac{z}{z_{3}} & =\frac{z_{4} \cos \left(k_{3} l\right)-i z_{3} \sin \left(k_{3} l\right)}{z_{3} \cos \left(k_{3} l\right)-i z_{4} \sin \left(k_{3} l\right)} \\
& =\frac{z_{4}\left\{1-i\left(z_{3} / z_{4}\right) \tan \left(k_{3} l\right)\right\}}{z_{3}\left\{1-i\left(z_{4} / z_{3}\right) \tan \left(k_{3} l\right)\right\}}
\end{aligned}
$$

Here

$$
z_{3}=\sqrt{\frac{-i \omega \mu_{3}}{\sigma_{3}-i \omega \varepsilon_{3}}} \quad, \quad k_{3}=\sqrt{i \omega \mu_{3}\left(\sigma_{3}-i \omega \varepsilon_{3}\right)}
$$

Equation (4.6) can be simplified in various limiting cases. For example, if

1) $\left|k_{3} l\right|<1$

$$
\begin{aligned}
\frac{z}{z_{4}} & \simeq 1-i k_{3} \ell\left(\frac{z_{3}}{z_{4}}-\frac{z_{4}}{z_{3}}\right) \\
& \simeq 1-i \ell\left\{\frac{\omega \mu_{3}}{z_{4}}-z_{4}\left(\omega \varepsilon_{3}+i \sigma_{3}\right)\right\}
\end{aligned}
$$

2) $\sigma_{3} \gg \omega \varepsilon_{3}$

$$
\frac{z}{z_{4}} \approx \frac{1-\frac{(i+1) \omega \mu_{3} \delta_{3}}{2 Z_{4}} \tan \left(\frac{1+i}{\delta_{3}} l\right)}{1+\frac{(1-i) Z_{4}}{\omega \mu_{3} \delta_{3}} \tan \left(\frac{1+i}{\delta_{3}} l\right)}
$$

where $k_{3} \simeq(1+i) / \delta_{3}, \quad z_{3} \simeq \frac{1-i}{2} \omega_{3} \delta_{3}, \quad \delta_{3}{ }^{2}=\frac{2}{\omega \mu_{3} \sigma_{3}}$ 
In the general case. Eq. (4.6) can be used to calculate the effect of the coating.

IV. Energy Loss

For a beam bunch with longltudinal charge density

$$
f(z)=\sum_{n=-\infty}^{\infty} f_{n} e^{i n z / R}, f_{n}=\frac{1}{2 \pi R} \int d z e^{-i n z / R} f(z)
$$

one has for the harmonic distribution of the circulating current

$$
I(z, t)=I_{0} \sum_{n=-\infty}^{\infty} \frac{f_{n}}{f_{0}} e^{i n(z-v t) / R}
$$

The energy loss per turn, for a particle located at $z-v t=\xi$ relative to the center of the bunch, is

$$
\begin{gathered}
\Delta W=e \operatorname{Re} \int_{0}^{2 \pi R} d z E_{z}(z, t=(z-\xi) / v) \\
(\operatorname{Re} \equiv \text { real part ) }
\end{gathered}
$$

since the coupling impedance is defined by the Fourier decomposition of $E_{z}(z, t)$ as

$$
2 \pi R E_{z}(z, t)=I_{0} \sum_{n=-\infty}^{\infty} Z_{L}(n) \frac{f_{n}}{f_{0}} e^{i n(z-v t) / R}
$$

we obtain

$$
\Delta W(\xi)=e I_{0} \quad \sum_{n=-\infty}^{\infty} \frac{f_{n}}{f_{0}} \operatorname{Re}\left\{Z_{L}(n) e^{i n \xi / R}\right\}
$$


which is close to Ruggiero's result (FN-230 (7)).

since we have $h$ bunches in the $r$ ing, where $h$ is the harmonic number, we have

$$
f(z)=\sum_{j=1}^{h} \rho\left(z-\frac{2 \pi R}{h} j\right)
$$

where $p$ is the density distribution of a single bunch. We therefore have, from Eq. (5.1),

$$
f_{n}=\frac{1}{2 \pi R} \int d z \rho(z) e^{-i n z / R} \sum_{j=1}^{h} e^{-i \frac{2 \pi j n}{h}}
$$

Since

$$
\underbrace{h}_{-\sum=1} e^{-i 2 \pi j n / h}=\left\{\begin{array}{l}
h, \quad n=0, \pm h, \pm 2 h, \ldots \\
0, \text { all other } n
\end{array}\right.
$$

we have

$$
\frac{f n}{f_{0}}=\left\{\begin{array}{cl}
\frac{\int d z \rho(z) e^{-i n z / R}}{\int d z \rho(z)}, & n=0, \pm h, \pm 2 h, \ldots \\
0, & \text { all other } n
\end{array}\right.
$$

and the sum in Eq. (5.5) only goes over integral values of $n / h$. For a beam bunch of length \&, the cutoff in $n$ equivalent to Eq. (5.1) is

$$
n_{0} \sim 2 \pi R / \ell
$$


Using the estimates of coupling impedance in Eq. (3.16), and $\mathrm{n}_{0} \sim 1000$, one obtains an energy loss of the order $100 \mathrm{keV}$ per turn at the center of the bunch which is in agreement with that estimated by Ruggiero (FN-230).

The procedure outlined above should be valld as long as the r.f. wave length. Is longer than the width of the laminations if they extend all around the circumference. The situation is less clear for wave lengths shorter than Individual magnet lengths, but we will accept Rugglero's and Snowdon's concept of a "filling factor" $\hat{\alpha}$, as contained in Eqs. (3.15) and (3.16).

As a final comparison, Ruggiero's result for the energy loss per particle per turn is

$$
\Delta \hat{w}(\xi)=N e^{2} B C \quad \sum_{n=0}^{\infty} \frac{f_{n}}{f_{0}} \operatorname{Re}\left\{\hat{Z}_{L}(n) e^{i n \xi / R}\right\}
$$

where $\hat{z}_{L}(n)$ corresponds to his definition of impedance. Using Ruggiero's asymptotic value $\operatorname{Re} \hat{Z}_{L}(n)=3.5 \times 10^{-13}$, with $N=4.2 \times 10^{10}$ protons per bunch, one obtains for $\beta=1$,

$$
\Delta \hat{W}(\xi) \simeq 60 \mathrm{eV} \sum_{n=0} \frac{f_{n}}{f_{0}} e^{i n \xi / R}
$$

Our analogous result for $I_{0}=335 \mathrm{~mA}, \hat{\alpha}=.6$, is given in Eqs. (5.5) and (3.16):

$$
\Delta W(\xi) \simeq 8 \mathrm{keV} \sum_{n=0}^{\infty} \frac{f_{n}}{f_{0}} e^{i n \xi / R}
$$


where the sum over $n$ in Eq. (5.13) includes only multiples of $h$, the harmonic number. Assuming a smooth variation of $f_{n}$ with n. Eq. (5.13) can be rewritten as

$$
\Delta W(\xi) \simeq 95 \mathrm{eV} \sum_{\text {ali }}^{\infty} \frac{f_{n}}{f_{0}} e^{i n \xi / R}
$$

in reasonable agreement with Ruggiero. For $n_{0} \sim 1000$, the energy los is of order 50 to $100 \mathrm{keV}$ per particle per turn. 
V. References

1. S.C. Snowdon, Wave Propagation Between Booster

Laminations Induced by Longitudinal Motion of Beam. TM-277, November 3, 1970.

2. A.G. Ruggiero, Longitudinal Space Charge Forces Within Bunched Beams, FN-219, December 15, 1970; Longitudinal Space Charge Forces Within a Bunched Beam in the Presence of Magnetic Laminations, FN-220, January 1971; Energy Loss Due to the Resistive Magnet Lamination in the FNAL Booster. FN230. May 25, 1971.

3. See, for example, Nielsen, Sessler and Symon, Proc. of the International Conference on High Energy Accelerators, Geneva, 1959, p. 239; A.W. Chao. 1982 Summer School Lectures, SLAC, p. 396.

4. R.L. Gluckstern, Longitudinal Coupling Impedance for a Cavity and Beam Pipe, TM-1268, June 1984.

5. Some authors give a result where the $1 / 4$ is replaced by 1/2. The result in Eq. (2.5) comes from averaging the electric field over the beam cross section, rather than taking its value at $r=0$. 